\title{
152. On Isometric Analytic Functions in Abstract Spaces
}

\author{
By Isae SHIModA \\ Mathematical Institute, Gakugei College, Tokushima University \\ (Comm. by K. Kunugi, M.J.A., Oct. 12, 1954)
}

In classical analysis, if a complex valued function $f(z)$ is regular and satisfies $|f(z)|=|z|$ in a circle $|z|<R$ in complex plane, then $f(z)=e^{i \theta} z$, that is, $f(z)$ is linear. We expect that an isometric analytic function is also linear in complex Banach spaces. An isometric analytic function is linear in fact if it is analytic on whole spaces but is not necessarily linear if it is defined on a bounded domain in complex Banach spaces.

Let $E, E^{\prime}$ be complex Banach spaces and $\alpha, \beta$ be complex numbers. An $E^{\prime}$-valued function defined on a domain $D$ in $E$ is analytic $^{1.2)}$ in $D$, if it is continuous strongly and admits Gateaux differential in $D$. An analytic function $h_{n}(x)$ which satisfies $h_{n}(\alpha x)$ $=\alpha^{n} h_{n}(x)$ for an arbitrary complex number $\alpha$ and an arbitrary point $x$ in $E$ is called a homogeneous polynomial of degree $n .^{37}$

Theorem 1. If an $E^{\prime}$-valued function $f(x)$ is analytic in the sphere $\|x\|<R$ in $E$ and satisfies $\|f(x)\| \leq K\|x\|^{m}$, then

$$
f(x)=\sum_{n=m}^{\infty} h_{n}(x)
$$

where $R, K$ and $m$ are constants and $h_{n}(x)$ is a homogeneous polynomial of degree $n$.

Proof. Since $f(x)$ is analytic in the sphere $\|x\|<R$, we have

$$
f(x)=\sum_{n=0}^{\infty} h_{n}(x)
$$

where $h_{n}(x)$ is a homogeneous polynomial of degree $n$ and

$$
h_{n}(x)=\frac{1}{2 \pi i} \int_{c} \frac{f(\alpha x)}{\alpha^{n+1}} d \alpha,
$$

for $n=1,2,3, \ldots$ and $C$ is a circle $|\alpha|=r$ such that $1<r<\frac{R}{\|x\|}$

Then, we have

$$
\begin{aligned}
\left\|h_{n}(x)\right\| & \leq\left\|\frac{1}{2 \pi i} \int_{c} \frac{f(\alpha x)}{a^{n+1}} d \alpha\right\| \leq \frac{1}{2 \pi} \int_{0}^{2 \pi} \frac{\left\|f\left(r e^{i \theta} x\right)\right\|}{r^{n}} d \theta \\
& \leq \frac{1}{2 \pi} \int_{0}^{2 \pi} \frac{K \|\left. r e^{i \theta} x\right|^{m}}{r^{n}} d \theta=K r^{m-n} \|\left. x\right|^{m},
\end{aligned}
$$

since $\|f(\alpha x)\| \leq K\|\alpha x\|^{m}$ for $\|\alpha x\|<R$. Since $r$ can be taken as close as we like to 1 , we have $\left\|h_{n}(x)\right\| \leq K|| x \|^{m}$, for $\|x\|<R$. Clearly, $\|\beta x\|$ $<R$ if $|\beta|<1$, and we have $\left\|h_{n}(\beta x)\right\| \leq K|| \beta x \|^{m}$. Then, $|\beta|^{n}|| h_{n}(x) \|$ $\leq K|\beta|^{m}|| x||^{m}$. If $n<m,\left\|h_{n}(x)\right\| \leq\left. K||^{m-n}|| x\right|^{m}$, for $0<|\beta| \leq 1$. Let $\beta$ 
tend to zero and we have $\left\|h_{n}(x)\right\|=0$ for an arbitrary point in the sphere $\|x\|<R$. Thus we can easily see that $h_{n}(x) \equiv 0$ for $n<m$. This completes the proof.

Theorem 2. If an $E^{\prime}$-valued function $f(x)$ defined in a sphere $\|x\|<R$ in $E$ is analytic and satisfies $\|f(x)\|=\|x\|^{m_{3}}$, then $f(x)=\sum_{n=m}^{\infty} h_{n}(x)$ and $\|f(x)\|=\left\|h_{m}(x)\right\|=\|x\|^{m}$.

Proof. Appealing to Theorem 1, we have $f(x)=\sum_{n=m}^{\infty} h_{n}(x)$. Since $\|\alpha x\|<R$ for an arbitrary point $x$ in a sphere $\|x\|<R$ and $|\alpha|<1$, we have $\|f(\alpha x)\|=\|\alpha x\|^{m}=|\alpha|^{m} \cdot\|x\|_{0}^{m} \quad$ On the other hand, $\|f(\alpha x)\|=$ $\left\|\sum_{n=m}^{\infty} h_{n}(x) \alpha^{n}\right\|=|\alpha|^{m}\left\|\sum_{n=m}^{\infty} h_{n}(x) \alpha^{n-m}\right\|$. Then, we have $\|x\|^{m}=\left\|\sum_{n=m}^{\infty} h_{n}(x) \alpha^{n-m}\right\|$, for $0<|\alpha|<1$. Let $\alpha$. tend to zero, we have $\|x\|^{m}=\left\|h_{m}(x)\right\|$. Since $x$ is an arbitrary point in the sphere $\|x\|<R$, we can easily have $\left\|h_{m}(x)\right\| \equiv\|x\|^{m}$.

Especially, for $m=1$, we have the following Corollary.

Corollary. If an $E^{\prime}$-valued function $f(x)$ defined in a sphere $\|x\|<R$ in $E$ is analytic and isometric, then $f(x)=\sum_{n=1}^{\infty} h_{n}(x)$ and $\|f(x)\|$ $=\left\|h_{1}(x)\right\|=\|x\|$.

In the same way, we can prove the following modified extended Liouville's theorem. ${ }^{144}$

Theorem 3. Let $E^{\prime}$-valued function $f(x)$ be an analytic function defined for all finite values in $E$. If $\|f(x)\| \leq 0\left(\|x\|^{m}\right)$ as $\|x\| \rightarrow \infty$, $f(x)$ is a polynomial of degree $m$ at most.

From Theorems 1 and 3, we can easily have following theorem.

Theorem 4. If an $E^{\prime}$-valued function $f(x)$ is analytic on whole space $E$ and satisfies $\|f(x)\| \leq K|| x \|^{m}$ for all finite values in $E$, then $f(x)$ is a homogeneous polynomial of degree $m$.

Corollary. If an $E^{\prime}$-valued function $f(x)$ defined on whole points in $E$ is analytic in $E$ and satisfies $\|f(x)\|=\|x\|^{m}$, then $f(x)$ is a homogeneous polynomial of degree $m$. Especially, if $\|f(x)\|=\|x\|, f(x)$ is linear.

Thus we see that an $E^{\prime}$-valued function $f(x)$ is linear if it is analytic and isometric on whole space, but $f(x)$ is not necessarily linear and satisfies only $\|f(x)\|=\left\|h_{1}(x)\right\|$, if it is isometric and analytic in a bounded domain as Corollary of Theorem 2 and following examples show.

Let $\Omega$ be complex- $D_{w}$-spaces. Put

$$
f(z)=\left(z\left(\frac{N+z}{2 N}\right), z\left(\frac{N+z}{2 N}\right)^{\frac{1}{2}}, \ldots, z\left(\frac{N+z}{2 N}\right)^{\frac{1}{n}}, \ldots\right),
$$

which is an $\Omega$-valued function defined in a circle $|z|<N$ in complex plane, where $\left(\frac{N+z}{2 N}\right)^{\frac{1}{n}}$ is considered on one branch of its Riemann 
surfaces. Then $f(z)$ is a one-valued analytic function and

$$
\|f(z)\|=\operatorname{Sup}_{n \geq 1}|z| \cdot\left|\frac{N+z}{2 N}\right|^{\frac{1}{n}}=|z| \cdot \operatorname{Sup}_{n \geq 1}\left|\frac{N+z}{2 N}\right|^{\frac{1}{n}}=|z| \cdot
$$

Moreover, $\left\|h_{1}(z)\right\|=|z|$, since $h_{1}(z)=\left(\frac{z}{2}, \underset{2}{z}, \ldots, \sqrt[m]{2}, \ldots\right)$. That is, $f(z)$ is isometric and analytic in $|z|<N$ and yet is non-linear.

Put $g(z)=\left(z, z^{2}, \ldots, z^{n}, \ldots\right)$,

which is an $\Omega$-valued function defined in the circle $|z|<1$ in complex plane. Then $g(z)$ is analytic in $|z|<1$ and satisfies ||$g(z)||=|z|$ and yet is not linear. Since $\|g(z)\|=+\infty$ if $|z|>1,|z|=1$ is a natural boundary of a domain $|z|<1$ in which $g(z)$ is analytic and isometric.

Put $h(z)=\left(z, z^{2}, \ldots, z^{n}, 0,0,0, \ldots\right)$,

which is an $\Omega$-valued polynomial of degree $n$ defined in complex plane. Then

$$
\begin{aligned}
\|h(z)\| & =|z|, & & \text { when }|z| \leq 1, \\
& =|z|^{n}, & & \text { when }|z| \geq 1 .
\end{aligned}
$$

That is, $h(z)$ is isometric and analytic in a circle $|z|<1$ and $\|h(z)\|$ $=O\left(|z|^{n}\right)$ as $|z| \rightarrow+\infty$. These examples shows us that there exist analytic functions which are added to homogeneous polynomials without changing their norms.

\section{References}

1) A.E. Taylor: Analytic function in general analysis, Annali della R. Scuola Normale Superiore di Pisa, Seri., 6, 15 (1937).

2) E. Hille: Functional analysis and semi-groups.

3) I. Shimoda: Notes on general analysis (II), Jour. Gakugei, Tokushima Univ., 3.

4) I. Shimoda: Notes on general analysis (III), On the norm of analytic functions, Jour. Gakugei, Tokushima Univ., 4, 2 (1954). 